\title{
A Model Study of Downward Walking Slip and Fall Probability Prediction Based on Coefficient of Friction Analysis
}

\author{
Junxia Zhang \\ College of Mechanical Engineer \\ TianJin University of Science and Technology \\ TianJin, China \\ zjx@tust.edu.cn \\ Hailong $\mathrm{Su}$ \\ College of Mechanical Engineer \\ TianJin University of Science and Technology \\ TianJin, China \\ suhailong@tust.edu.cn
}

\author{
$\mathrm{Na}$ Yin \\ College of Mechanical Engineer \\ TianJin University of Science and Technology \\ TianJin, China \\ yinna@tust.edu.cn \\ Weiwei Wang \\ College of Mechanical Engineer \\ TianJin University of Science and Technology \\ TianJin, China \\ wangweiweibgfe@yahoo.com.cn
}

\begin{abstract}
In view of there is a room for improvement for the accuracy in using the slip and fall judgment condition proposed in early time, in addition, slips and falls are easier to happen at the time of downward walking, so, through orthogonal tests in terms of sole material, ground material, contaminated medium and slope grade, a great deal of data has been obtained for analyzing relations of ACOF respectively with RCOF and UCOF in downward walking stability. On this basis, this paper also further optimize the slip and fall probability prediction model, making them apply to cases of downward walking and achieving more accurate effects.
\end{abstract}

Keywords- Slips and falls; Prediction model; Downward walking; Coefficient of friction

\section{INTRODUCTION}

Slip and fall have been commonly seen in daily life, industrial production and other areas. In accordance with statistics, 391,000 deaths were due to slips and falls around the world in 2003 [1]. As follows are introduced some coefficients of friction related to slips and falls.

Available Coefficient of Friction (ACOF) means the maximum coefficient of friction, provided between the sole and the ground when there is no slipping. Required Coefficient of Friction (RCOF) means the maximum ratio of horizontal resultant force and vertical resultant force of sole-ground contact force when a person is walking on a dry ground. All the aforesaid coefficients of friction are calculated on dry surfaces. This penal has proposed the concept of Utilized Coefficient of Friction (UCOF) means the maximum values of horizontal resultant force and vertical resultant force of sole-ground contact force at the time of walking on a ground with contaminated medium [2].

As is known, the risk of slips and falls when walking

The project is supported by China Natural Science Foundation (50975204) downward is higher than that when walking on a flat ground. The result of $\mathrm{Li}$ et al shows that the cosine function of slope grade is used to express relations between coefficients of friction under conditions of sloping and flat ground [3]. This panel's research show that, as the slope grade increases, the RCOF and the UCOF increase and proposed a new condition: if RCOF > ACOF, a slip and fall will happen; if UCOF > ACOF, the person will continue slipping and fall [2].

\section{TEST METHOD}

\section{A. Test Subject and Equipment}

The subjects of this research respectively are five 25 -yearold healthy men and women. Test equipments mainly include walking test bench, accompanying protective device and data collection system. The walking test bench is designed with two parts, with one part's end able to be freely adjusted within the range of $0^{\circ} \sim 15^{\circ}$ to obtain a required slope angle. Two force plates are placed in the middle (Figure 1). The data collection system comprises two force platforms, six MX infrared cameras, Vicon Vegas converter, PC main frame, and Vicon Datastation ADC Patch Panel information boxcar. The force platform has a collection frequency of $1000 \mathrm{~Hz}$ and connects to the computer through Vicon Vegas converter. MX cameras are used to record three-dimensional data of lower limbs when a person walks and are distributed on both sides of the track, all over the force platforms. Camera arrangement as shown in Figure 2. 


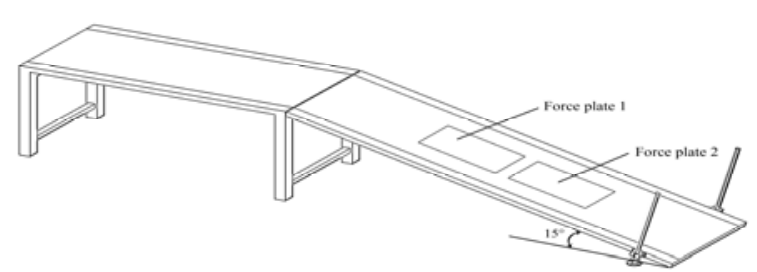

Figure 1 walking test bench

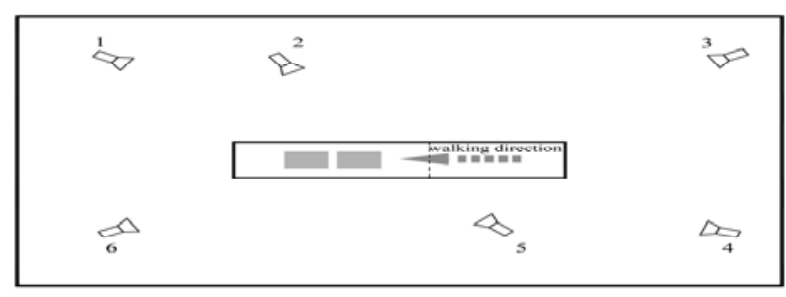

Figure2 Camera arrangement in level slips and falls trail

\section{B. Test Design}

The conditions of this test: sole material is Natural rubber; ground material is Marble; contaminated medium are dry and vegetable oil; Slope grade are $0^{\circ}, 5^{\circ}, 10^{\circ}$ and $15^{\circ}$.In order to make the subjects well finish the test, they will be informed of the test objective, content and matters for attention prior to the test. Then, they will be arranged to walk on the test bench for times to ensure effective test data is obtained. In the test, RCOF and UCOF will be repeatedly collected with the same condition and data will be recorded. The subjects will get a break between two combination tests.

\section{TEST RESUlt AND ANALysis}

\section{A. Optimization of Dry Ground Slip Probability Prediction Model}

This paper uses No.1 subject to do gait test with the combination of natural rubber-marble-dry- $0^{\circ}$ conditions to define the dry flat ground slip condition. No.1 subject is used to respectively do gait test with combinations of natural rubber-marble-dry- $5^{\circ} / 10^{\circ} / 15^{\circ}$ conditions to define the dry sloping ground slip condition.

For No.1 subject doing the gait test with the combination of natural rubber-marble-dry- $0^{\circ}$ conditions, the test is done for 120 times, and corresponding RCOF is recorded. Early research findings of this panel show under this combination of conditions, $\mathrm{ACOF}=0.208$ [2], and all the RCOF of 117 times among the 120 times are greater than ACOF under this combination of condition. In accordance with the slip judgment condition proposed by this panel in early time: If RCOF $>$ ACOF, a slip will happen, then all these 117 times of gait test should see a slip, but the test results show only 75 times among the 120 times of gait test see slip, with others seeing no slipping phenomenon and the subject having no feel of slipping. There is some difference between theory and reality, so the judgment condition needs correction to improve accuracy.
It is assumed the dry flat ground slip judgment condition is: If $\mathrm{RCOF} \geq \mathrm{a}^{*} \mathrm{ACOF}$ ( $\mathrm{a}$ is a constant greater than 1 ), a slip will happen. In accordance with statistics, the minimum corresponding RCOF in the 75 times of gait test seeing a slip is 0.482778 ; at that time, $\mathrm{RCOF}=2.321 * \mathrm{ACOF}$, so the dry flat ground slip judgment condition can be corrected into: If RCOF $\geq 2.321 *$ ACOF, a slip will happen.

For No.1 subject doing the gait test with the combination of natural rubber-marble-dry $-5 \% / 10^{\circ} / 15^{\circ}$ conditions, 150 times of downward walking test are done, and corresponding RCOF is recorded. The research findings of Chang WR et al [3] show: $A C O F_{\text {Downward walking }}=\cos \theta^{*} A C O F_{\text {Flat ground }}$, in accordance with this research result, ACOF for $5^{\circ}, 10^{\circ}$ and $15^{\circ}$ is respectively calculated, as shown in Table 1.

TABLE I. $\quad A C O F_{\text {Downward walking }}$ FOR $5^{\circ}, 10^{\circ}$ AND $15^{\circ}$

\begin{tabular}{|c|c|c|}
\hline Slope Grade $\boldsymbol{\theta}$ & $\cos \boldsymbol{\theta}$ & ACOF $_{\boldsymbol{\theta}}$ \\
\hline $5^{\circ}$ & 0.996195 & 0.207209 \\
\hline $10^{\circ}$ & 0.984808 & 0.204840 \\
\hline $15^{\circ}$ & 0.965926 & 0.200913 \\
\hline
\end{tabular}

Among the 50 times of downward walking test with a slope grade of $5^{\circ}$, all corresponding RCOF is greater than ACOF under this condition. In accordance with the slip and fall judgment condition proposed by this panel in early time, all the 50 times of walking test should see a slip, but the test results show only in 38 times the subject has a feel of slipping, and the minimum corresponding RCOF for the 38 times of slip is 0.479206 , so the downward walking slip judgment condition with a slope grade of $5^{\circ}$ is: If RCOF $\geq 2.312 * \mathrm{ACOF}$, a slip will happen. Likewise, the downward walking slip judgment conditions with a slope grade of $10^{\circ}$ and $15^{\circ}$ can be obtained, as shown in Table 2.

TABLE II. SLIP JUDGMENT CONDITIONS ON DRY SURFACE

\begin{tabular}{|c|c|c|c|}
\hline $\begin{array}{c}\text { Slope } \\
\text { Grade }\end{array}$ & $\begin{array}{c}\text { Walking } \\
\text { Direction }\end{array}$ & $\begin{array}{c}\text { The Minimum For } \\
\text { The 38 Times Of Slip }\end{array}$ & $\begin{array}{c}\text { Slip Judgment } \\
\text { Conditions }\end{array}$ \\
\hline $0^{\circ}$ & $/$ & 0.208000 & \multirow{2}{*}{$\operatorname{RCOF}_{\theta} \geq 2.321 \cos \theta^{*} \mathrm{ACOF}_{\theta}$} \\
\hline $5^{\circ}$ & $\begin{array}{c}\text { downward } \\
\text { walking }\end{array}$ & 0.207209 & \\
\cline { 1 - 3 } $10^{\circ}$ & $\begin{array}{c}\text { downward } \\
\text { walking }\end{array}$ & 0.204840 & \\
\hline $15^{\circ}$ & $\begin{array}{c}\text { downward } \\
\text { walking }\end{array}$ & 0.200913 & \\
\hline
\end{tabular}

The judgment conditions in this paper and proposed by this panel in early time are used to respectively judge No.2 subject's slip situations with combinations of natural rubbermarble-dry- $0^{\circ} / 5^{\circ} / 10^{\circ} / 15^{\circ}$ conditions, and all theoretical judgment results and actual test results are shown in Table 3 . In comparison with the judgment condition proposed by this panel in early time, the dry ground slip judgment condition in this paper sees some improvement in accuracy. 
TABLE III. THE CONTRAST RESULT OF NO.2 SUBJECT'S SLIP SITUATIONS

\begin{tabular}{|c|c|c|c|c|}
\hline \multirow{2}{*}{$\begin{array}{c}\text { Slope } \\
\text { Grade }\end{array}$} & $\begin{array}{c}\text { Actual } \\
\text { Slip } \\
\text { Times }\end{array}$ & \multirow{2}{*}{$\begin{array}{c}\text { Slip Judgment } \\
\text { Conditions }\end{array}$} & $\begin{array}{c}\text { Theoretical } \\
\text { Slip Times } \\
\mathbf{2}\end{array}$ & $\begin{array}{c}\text { The Range } \\
\text { Of } \\
\text { Improvement } \\
\text { In Accuracy }\end{array}$ \\
\hline $0^{\circ}$ & 0.208000 & & 118 & 83 \\
\hline $5^{\circ}$ & 0.207209 & \multirow{2}{*}{$\operatorname{RCOF}_{\theta} \geq 2.321 \cos \theta^{*} \mathrm{ACOF}_{\theta}$} & 83 & $30.2 \%$ \\
\cline { 1 - 1 } & & & 42 & $14.9 \%$ \\
\hline $10^{\circ}$ & 0.204840 & & 44 & $11.5 \%$ \\
\hline
\end{tabular}

Note: Theoretical Slip Times 1From The Judgment Condition Proposed By This Panel In Early Time, Theoretical Slip Times 2 From The Judgment Condition In This Paper.

Because in early analyses of flat ground slip and fall data, this panel has used many methods to prove ACOF and RCOF under the same combination of conditions all display a normal distribution [2], the probability density functions of $R C O F_{\theta}$ and $A C O F_{\theta}$ under the same combination of conditions with a fixed slope grade respectively are Formula 1 and Formula 2:

$$
\begin{aligned}
& P_{r(\theta)}\left(\mu_{r(\theta)}\right)=\frac{1}{\sqrt{2 \pi} \sigma_{r(\theta)}} e^{-\frac{1}{2}\left(\frac{\mu_{u(\theta)}-\bar{\mu}_{r(\theta)}}{\sigma_{r(\theta)}}\right)^{2}} \\
& P_{a(\theta)}\left(\mu_{a(\theta)}\right)=\frac{1}{\sqrt{2 \pi} \sigma_{a(\theta)}} e^{\left.-\frac{1}{2} \frac{\mu_{a(\theta)}-\bar{\mu}_{a(\theta)}}{\sigma_{a(\theta)}}\right)^{2}}
\end{aligned}
$$

wherein, $\bar{\mu}_{r(\theta)}$ and $\sigma_{r(\theta)}$ respectively represent the expectancy and variance of $R C O F_{\theta}, \bar{\mu}_{a(\theta)}$ and $\sigma_{a(\theta)}$ respectively represent the expectancy and standard deviation of $A C O F_{\theta}$. Because the linear combination of both functions conforming to normal distribution still conforms to normal distribution, the difference between $R_{C O F}$ and $A C O F_{\theta}$ still conforms to normal distribution, if $\mu_{\xi}^{\prime}=\mu_{r(\theta)}-\mu_{a(\theta)}$, then:

$$
P_{\mu_{\xi}^{\prime}}\left(\mu_{\xi}^{\prime}\right)=\frac{1}{\sqrt{2 \pi} \sigma_{\xi}^{\prime}} e^{-\frac{1}{2}\left(\frac{\mu_{\xi}^{\prime}-\bar{\mu}_{\xi}^{\prime}}{\sigma_{\xi}^{\prime}}\right)^{2}}
$$

wherein, $\bar{\mu}_{\xi}^{\prime}=\bar{\mu}_{r(\theta)}-\bar{\mu}_{a(\theta)}, \sigma_{\xi}^{\prime}=\sqrt{\sigma_{r(\theta)}^{2}+\sigma_{a(\theta)}^{2}}$

Therefore, the dry ground slip probability prediction model is obtained as below:

$$
P\left(\mu_{\xi}^{\prime} \geq 0\right)=\int_{1.321 A C O F_{\theta}}^{+\infty} \frac{1}{\sqrt{2 \pi} \sigma_{\xi}^{\prime}} e^{-\frac{1}{2}\left(\frac{\mu_{\xi}^{\prime}-\vec{\mu}_{\xi}^{\prime}}{\sigma_{\xi}^{\prime}}\right)^{2}} d \mu_{\xi}^{\prime}
$$

B. Optimization of Contaminated Medium Ground Slip and Fall Probability Prediction Model

This paper uses No.1 subject to do gait test with the combination of natural rubber-marble-oil- $0^{\circ}$ conditions to define the contaminated medium flat ground slip and fall judgment condition. No.1 subject is used to do gait test with the combination of natural rubber-marble-oil- $5^{\circ}$ conditions to define the sloping ground slip and fall condition.

For No.1 subject doing the gait test with the combination of natural rubber-marble-oil- $0^{\circ}$ conditions, the test is done for 120 times, and corresponding UCOF is recorded. Early research findings of this panel show under this combination of conditions, $\mathrm{ACOF}=0.033$ [2], and in accordance with the slip and fall judgment condition proposed therein: If UCOF > ACOF, a slip will continue and a fall will happen. All the UCOF of the 150 times are greater than ACOF under this combination of condition; in accordance with the above slip and fall judgment condition, these 150 times of gait test should see a continuous slip and a fall, but the test results show only 106 times among the 150 times of gait test see a continuous slip and a fall, with others seeing a slip and a balance recovery. There is some difference between theory and reality, so the contaminated medium flat ground slip and fall judgment proposed by this panel needs correction to improve accuracy. It is assumed the contaminated medium flat ground slip and fall judgment condition is: If $\mathrm{RUCOF}<\mathrm{b}^{*} \mathrm{ACOF}$, a slip will happen; if $\mathrm{UCOF} \geq \mathrm{b}^{*} \mathrm{ACOF}$, a slip will continue and a slip will happen, wherein $b$ is a constant greater than 1 . In accordance with statistics, the minimum corresponding UCOF in the 106 times of gait test seeing a fall is 0.112647 ; at that time, $\mathrm{UCOF}=3.414 * \mathrm{ACOF}$, so the contaminated medium flat ground slip and fall judgment condition can be corrected into: If $\mathrm{ACOF}<\mathrm{UCOF}<3.414^{*} \mathrm{ACOF}$, a slip will happen; if $\mathrm{UCOF} \geq 3.414 * \mathrm{ACOF}$, a slip will continue and a fall will happen.

For No.1 subject doing the gait test with the combination of natural rubber-marble-oil- $5^{\circ}$ conditions, 50 times of downward walking test are done, and corresponding UCOF is as shown in Table 4.

TABLE IV. THE UCOF OF NO.1 SUBJECT DOING THE GAIT TEST WITH THE COMBINATION OF NATURAL RUBBER-MARBLE-OIL- $5^{\circ}$ CONDITIONS

\begin{tabular}{|c|c|c|c|c|}
\hline $\begin{array}{c}\text { Test } \\
\text { Number }\end{array}$ & \multicolumn{4}{|c|}{ UCOF } \\
\hline $1-5$ & 0.307425 & 0.437432 & 0.461047 & 0.527897 \\
\hline $6-10$ & 0.401865 & 0.343947 & 0.294302 & 0.371967 \\
\hline $11-15$ & 0.612039 & 0.104552 & 0.099856 & 0.205124 \\
\hline $16-20$ & 0.363504 & 0.567853 & 0.178522 & 0.533362 \\
\hline $21-30$ & 0.371458 & 0.335562 & 0.102145 & 0.421587 \\
\hline $31-40$ & 0.520300 & 0.478521 & 0.326533 & 0.412583 \\
\hline $41-50$ & 0.1102263 & 0.156953 & 0.433098 & 0.363252 \\
\hline
\end{tabular}

Time, Theoretical Slip Times 2 From The Judgment Condition In This Paper.

In accordance with the research findings of Chang WR et al [4], under the combination of natural rubber-marble-oil $5^{\circ}$ 
conditions, $\mathrm{ACOF}=0.032874$; in the 50 times of test with a slope grade of $5^{\circ}$, all corresponding UCOF is greater than ACOF under the corresponding condition. In accordance with the slip and fall judgment condition proposed by this panel in early time, all the 50 times of test should see a continuous slip and a fall, but the test results show: among the 50 times, 43 times see a continuous slip and a fall and 6 times see a slip and balance recovery, wherein the minimum UCOF in the 43 times of test seeing a continuous slip and fall is 0.111894 , while $\mathrm{UCOF}=3.404 * \mathrm{ACOF}$. So if the slope grade is $\theta$, the downward walking slip and fall judgment condition is specified as: If $A C O F_{\theta}<U C O F_{\theta}<3.414 \cos \theta * A C O F_{\theta}$, a slip will happen; if $U C O F_{\theta} \geq 3.414 \cos \theta * A C O F_{\theta}$, a slip will continue and a fall will happen. The other nine subjects conduct the test for 450 times in total under the combination of natural rubber-marble-oil- $5^{\circ}$ conditions, the slip and fall judgment condition proposed by this panel in early time and the judgment condition proposed in this paper are respectively used to make judgment, with results as shown in Table 5. The test results show that when judging the slip and fall situation of No.2 to No.10 subjects, the slip and fall judgment proposed in this paper sees an increase in accuracy in each case; because the test times are limited, this also affect the increase pace of accuracy to some extent.

TABLE 4 THE CONTRAST RESULT OF JUDGING THE SLIP AND FALL SITUATION OF NO. 2 TO NO. 10 SUBJECTS

\begin{tabular}{|c|c|c|c|c|c|c|c|}
\hline \multirow{2}{*}{$\begin{array}{l}\text { Subjects } \\
\text { Number }\end{array}$} & \multicolumn{2}{|c|}{$\begin{array}{l}\text { Actual } \\
\text { Times }\end{array}$} & \multicolumn{2}{|c|}{$\begin{array}{c}\text { Theoretical } \\
\text { Times } 1\end{array}$} & \multicolumn{2}{|c|}{$\begin{array}{c}\text { Theoretical } \\
\text { Times } 2\end{array}$} & \multirow{2}{*}{$\begin{array}{l}\text { The Range } \\
\text { Of } \\
\text { Improvement } \\
\text { In Accuracy }\end{array}$} \\
\hline & slip & fall & slip & fall & slip & fall & \\
\hline 2 & 10 & 40 & / & 50 & 8 & 42 & $15.2 \%$ \\
\hline 3 & 9 & 41 & / & 50 & 8 & 42 & $15.6 \%$ \\
\hline 4 & 11 & 39 & / & 50 & 9 & 41 & $17.1 \%$ \\
\hline 5 & 7 & 43 & / & 50 & 5 & 45 & $9.6 \%$ \\
\hline 6 & 8 & 42 & / & 50 & 7 & 43 & $13.7 \%$ \\
\hline 7 & 6 & 44 & / & 50 & 5 & 45 & $9.8 \%$ \\
\hline 8 & 9 & 41 & / & 50 & 7 & 43 & $13.3 \%$ \\
\hline 9 & 5 & 45 & / & 50 & 4 & 46 & $7.8 \%$ \\
\hline 10 & 11 & 39 & I & 50 & 8 & 42 & $14.9 \%$ \\
\hline
\end{tabular}

This panel has proved that ACOF and UCOF under the same combination of conditions all display a normal distribution [2], the probability density functions of $A C O F_{\theta}$ and $U C O F_{\theta}$ under the same combination of conditions with a fixed slope grade respectively are Formula 5 and Formula 6:

$$
P_{a(\theta)}\left(\mu_{a(\theta)}\right)=\frac{1}{\sqrt{2 \pi} \sigma_{a(\theta)}} e^{-\frac{1}{2}\left(\frac{\mu_{a(\theta)}-\bar{\mu}_{a(\theta)}}{\sigma_{a(\theta)}}\right)^{2}}
$$

$$
P_{u(\theta)}\left(\mu_{u(\theta)}\right)=\frac{1}{\sqrt{2 \pi} \sigma_{u(\theta)}} e^{-\frac{1}{2}\left(\frac{\mu_{u(\theta)}-\bar{\mu}_{u(\theta)}}{\sigma_{u(\theta)}}\right)^{2}}
$$

wherein, $\bar{\mu}_{a(\theta)}$ and $\sigma_{a(\theta)}$ respectively represent the expectancy and standard deviation of $\mathrm{ACOF}_{\theta} ; \bar{\mu}_{u(\theta)}$ and $\sigma_{u(\theta)}$ respectively represent the expectancy and standard deviation of $U C O F_{\theta}$. If $\mu_{\xi *}^{\prime}=\mu_{u(\theta)}-\mu_{a(\theta)}$, then the probability density function of $\mu_{\xi *}^{\prime}$ is:

$$
P_{\mu_{\xi^{*}}^{\prime}}\left(\mu_{\xi^{\prime}}^{\prime}\right)=\frac{1}{\sqrt{2 \pi} \sigma_{\xi^{*}}^{\prime}} e^{-\frac{1}{2}\left(\frac{\mu_{\xi^{*}-}^{\prime}-\bar{\mu}_{\xi^{*}}^{\prime}}{\sigma_{\xi^{*}}^{\prime}}\right)^{2}}
$$

$$
\text { wherein, } \bar{\mu}_{\xi *}^{\prime}=\bar{\mu}_{u(\theta)}-\bar{\mu}_{a(\theta)}, \sigma_{\xi *}^{\prime}=\sqrt{\sigma_{u(\theta)}^{2}+\sigma_{a(\theta)}^{2}}
$$

Therefore, the contaminated medium ground walking slip probability prediction model is

$$
P\left(\mu_{\xi^{*}}^{\prime} \geq 0\right)=\int_{0}^{(3.414 \cos \theta-1) A C O F_{\theta}} \frac{1}{\sqrt{2 \pi} \sigma_{\xi_{*}}^{\prime}} e^{-\frac{1}{2}\left(\frac{\mu_{\xi^{*}}^{\prime}-\vec{\mu}_{\xi^{*}}}{\sigma_{\xi^{*}}^{\prime}}\right)^{2}} d \mu_{\xi_{*}}^{\prime}
$$

The slip probability prediction model is:

$$
P\left(\mu_{\xi_{*}}^{\prime} \geq 0\right)=\int_{(3.414 \cos \theta-1) A C O F_{\theta}}^{+\infty} \frac{1}{\sqrt{2 \pi} \sigma_{\xi_{*}}^{\prime}} e^{-\frac{1}{2}\left(\frac{\mu_{\xi^{*}}^{\prime}-\bar{\mu}_{\xi_{*}}^{\prime}}{\sigma_{\xi_{*}}^{2}}\right.} d \mu_{\xi^{2}}^{\prime}
$$

\section{CONCLUSION}

The optimized slip and fall probability prediction models are better in conformity to daily life and working environment, not only applicable to flat ground walking, but also applicable to sloping ground walking, hence broadening their application scope. They can also more accurately predict slip and fall probabilities under specific conditions, providing a reference for the stability design of bipedal robots and mobility aids and a basis for their evaluation, providing a more reliable theoretical basis for making slip prevention strategies and designing anti-slipping devices, providing a guide for old people's daily walking, and providing a theoretical basis for studies in the areas of construction and sports etc.

\section{REFERENCES}

[1] WHO. The injury chartbook : a graphcal overview of the global burden of injury[R]. Geneva : WHO, $2002:$ 43-50.

[2] ZHANG Yunyun. A Model Study of Skid and Slip Probability Prediction Based on Human Gait Analysis [D]. TianJin : TianJin University Of Science And Technology, 2011.

[3] Li KW, Chang WR. Friction measurements on ramps using the Brungraber Mark slipmeter[J]. Safety Science, 2006, 44 : 375-386.

[4] Chang WR, Leclercq S, Brungraber R. The role of friction in the measurement of slipperiness, Part 2 : Survey of friction measurement devices[J]. Ergonomics, 44 (14) : 1233-1261. 